\title{
Sclerosing Mesenteritis: Multidisciplinary Collaboration Is Essential for Diagnosis and Treatment
}

\author{
Huan $\mathrm{He}^{\mathrm{a}}$, Min Zhi ${ }^{\mathrm{a}}$, e, Min Zhang ${ }^{\mathrm{a}}$, Mingli Su ${ }^{\mathrm{a}}$, Huangwei Chen ${ }^{\mathrm{a}}$, Liang Kang ${ }^{\mathrm{b}}$, \\ Yan Huang ${ }^{\mathrm{c}}$, Zhiyang Zhou ${ }^{\mathrm{d}}$, Xiang Gao ${ }^{\mathrm{a}}$, Jianping Wang ${ }^{\mathrm{b}}$, Pinjin $\mathrm{Hu}^{\mathrm{a}}$
}

\begin{abstract}
Sclerosing mesenteritis (SM) is an extremely rare disease characterized by chronic non-specific inflammation, fat necrosis and fibrosis of the mesentery. We presented a 77-year-old man with progressive dyschezia, abdominal pain and mass in left lower quadrant. Computed tomography (CT) exhibited a thickened mesentery, enlarged lymph nodes and strand-like densities around the mesenteric vessels. However, laboratory investigation, colonoscopy and positron emission tomography did not provide any specific results for diagnosis. Because of the exacerbating abdominal pain, partial colectomy was performed and SM was diagnosed based on the pathological changes of mesentery including fat necrosis, multifocal lipid-filled macrophages, lymphocytes and multifocal fibrosis. Although SM is difficult to diagnose and often found by incident, progressive deterioration of abdominal symptoms and general status alteration are indicators of SM. Some typical imaging and pathologic manifestations are also helpful to SM diagnosis. There is no standard treatment for SM. Operation is preferred in those at the stage of fibrosis and particularly combined with intestinal obstruction. Therefore, a multidisciplinary collaboration is essential to diagnose and manage this rare disease, with combined approaches in gastroenterology, colorectal surgery, pathology and radiology.
\end{abstract}

Keywords: Sclerosing mesenteritis; Computed tomography; Surgery; Histopathology; Multidisciplinary

\section{Introduction}

Sclerosing mesenteritis (SM) is a rare disease of uncertain eti-

Manuscript accepted for publication January 18, 2017

aDepartment of Gastroenterology, the Sixth Affiliated Hospital of Sun Yat-sen University, Guangzhou 510655, Guangdong Province China

bepartment of Colon and Rectum Surgery, the Sixth Affiliated Hospital of Sun Yat-sen University, Guangzhou 510655, Guangdong Province China

cDepartment of Pathology, the Sixth Affiliated Hospital of Sun Yat-sen University, Guangzhou 510655, Guangdong Province China

${ }^{\mathrm{d} D e p a r t m e n t ~ o f ~ I m a g e o l o g y, ~ t h e ~ S i x t h ~ A f f i l i a t e d ~ H o s p i t a l ~ o f ~ S u n ~ Y a t-s e n ~ U n i-~}$ versity, Guangzhou 510655, Guangdong Province China

${ }^{\mathrm{e} C}$ Corresponding Author: Min Zhi, Department of Gastroenterology, the Sixth Affiliated Hospital of Sun Yat-sen University, 26 Yuancun Erheng Road Tianhe District, the Sixth Affiliated Hospital of Sun Yat-sen University, Guangzhou 510655, Guangdong Province, China. Email: zhimin@mail.sysu.edu.cn

doi: https://doi.org/10.14740/gr732w ology characterized by chronic non-specific inflammation, fat necrosis and fibrosis involving adipose tissue of the mesentery. It was first described in the medical literature by Jura et al as "retractile mesenteritis" in 1924 [1]. In 1965, it was named as "mesenteric panniculitis" by Ogden et al [2]. SM is difficult to diagnose and often found accidently. Some typical imaging and histopathological manifestations are also helpful to SM diagnosis. Because SM is a rare disease, lacking the typical clinical presentation, the radiological and histopathological characteristics of mesentery is critical for diagnosis. There is not standard therapy. Pharmacotherapy and surgery are available to SM patients. Here we present a case of SM which was diagnosed based on the dominant pathological changes of mesentery obtained by surgery.

\section{Case Report}

A 77-year-old man, who was Han Chinese and retired, presented with progressive dyschezia, left lower quadrant abdominal pain and $1.5 \mathrm{~kg}$ weight loss within 3 months before admission to our hospital. Two weeks prior to his admission, the patient was admitted to a different hospital with aggravating abdominal pain. Laboratory investigation showed positive fecal occult blood, normal range of hemoglobin $(\mathrm{Hb})(12.4 \mathrm{~g} / \mathrm{dL})$ and Creactive protein (CRP) $(2.35 \mathrm{mg} / \mathrm{dL})$. Computed tomography (CT) revealed increased thickness of wall in multiple loops of small bowel, descending colon, ascending colon and rectum, multiple enlarged lymph nodes and strand-like densities around the mesenteric vessels (Fig. 1). Colonoscopy showed that the mucosa was diffuse hyperemia and edema from the descending colon to rectum, with some cobble stone sign. Mucosaliopsy showed non-specific chronic inflammation. He was treated for a presumed diagnosis of inflammatory bowel disease (IBD) with oral mesalazine (2.0 g QID) and intravenous dexamethasone (10 mg QD $\times 3$ days) but the patient's symptoms were not improved with these treatments. The patient was transferred to our hospital.

The patient denied recurrent fever, oral ulcer, arthralgia, ophthalmodynia, skin rash, cough, expectoration and night sweat. His past medical history included type 2 diabetes and hypertension for which he received oral hypoglycemic agents (dimethylbiguanide) and anti-hypertensives (captropril). He was allergic to cephalosporin. He had a recent trip to Russia, 1 month before the onset of symptoms. He also denied the history of trauma, surgery, blood transfusion, inherited diseases and psychiatric diseases. 


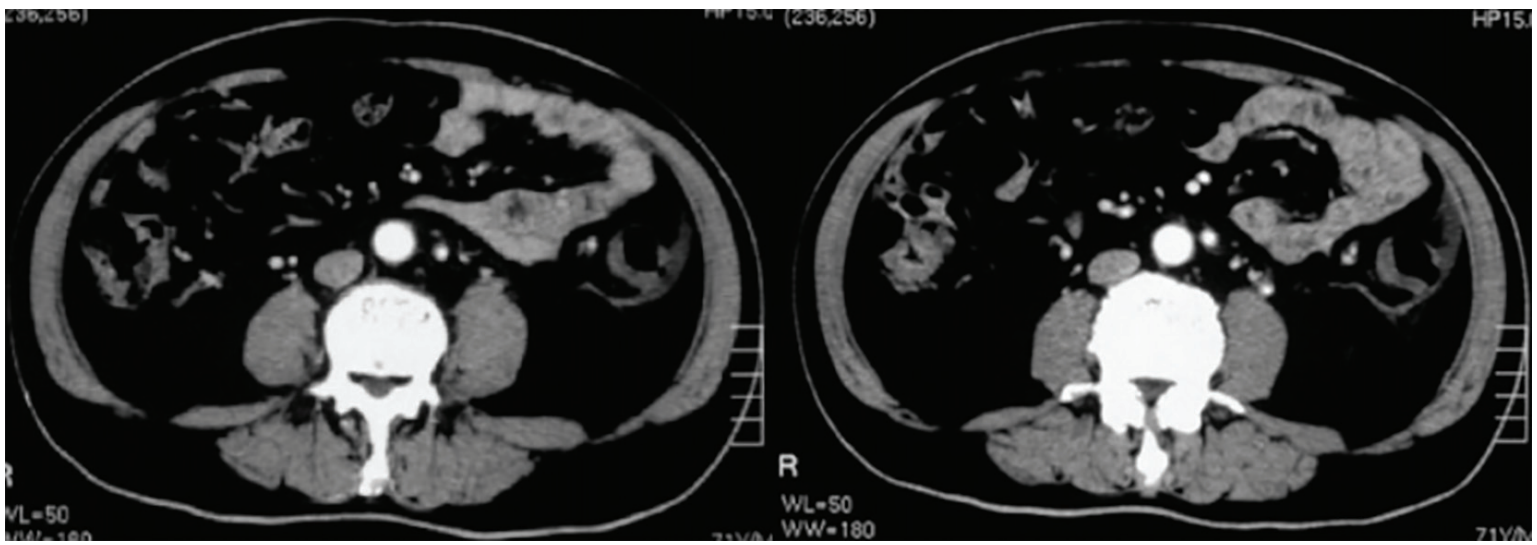

Figure 1. CT imaging of the abdomen showed thickening of the sigmoid colon wall which corresponded to a palpable firm cordlike mass $(20 \times 5 \mathrm{~cm})$ in the left lower quadrant with tenderness on physical examination.

Upon admission to our hospital, physical examination (PE) revealed tenderness on a palpable firm cord-like $20 \times 5$ $\mathrm{cm}$ mass in the left lower quadrant. No hepatosplenomegaly, lymphadenopathy, or skin rashes were noted. Digital rectal examination revealed a palpable tender nodule of the rectum mucosa from 7 to 9 o'clock at lithotomy position. Laboratory investigation in our hospital was notable for the high level of erythrocyte sedimentation rate $(44 \mathrm{~mm} / \mathrm{h}), \mathrm{CRP}(8.93 \mathrm{mg} / \mathrm{L})$, $\beta 2$-microglobulin $(1,883 \mu \mathrm{g} / \mathrm{L}(0-1,150))$, immunoglobulin (Ig) $\lambda$ chain $(17.2 \mathrm{~g} / \mathrm{L}(4.3-6.5 \mathrm{~g} / \mathrm{L}))$ and $\operatorname{IgG}(23.6 \mathrm{~g} / \mathrm{L}(7-15$ $\mathrm{g} / \mathrm{L})$ ), and for the normal range of hemoglobin, white blood cell count, neutrophil differential count, electrolytes, liver enzymes, bilirubin, and tumor markers.

The patient's esophagogastroduodenoscopy was normal. Colonoscopy demonstrated diffuse hyperemia, edema, and stiffness with cobble stone sign from the descending colon to rectum, with the rectum being most serious. There were circumferential erosions and two superficial ulcers in the rectum (Fig. 2). Histopathological evaluation of colon biopsy showed the mucosal infiltration by inflammatory cells and focal interstitial fibrosis (Fig. 3). Based on all available information, malignancy and inflammatory disease were the major differential diagnosis. The patient consented to endoscopic submucosal dissection (ESD) (Fig. 4) and colon biopsy in order to get large pathological tissue for diagnosis. Histopathology of specimen of the rectal ulcers from ESD showed erosion, inflammatory exudates, granulation tissue and fibrinoid degeneration of wall of small blood vessel (Fig. 5). Blood vessels and lymphatic vessels in the submucosa were dilated. Stromal edema was present. Because ESD did not provide evidences for specific diagnosis, we performed positron emission tomography (PET) on the patient. PET-CT showed significant accumulation of signal uptake, with diffuse edema and bowel wall thickening (about $1.5 \mathrm{~cm}$ ) in the distal descending colon, suggesting an inflammatory state (Fig. 6).

During his hospitalization, the patient had a worsened left lower quadrant abdominal pain accompanied by sweating. PE suggested increased tenderness in the left lower quadrant and suspicious rebound tenderness. Because of a suspicion for intestinal obstruction, an exploratory laparoscopy was performed. Intraoperatively, we found abdominal ascites of about $300 \mathrm{~mL}$, markedly thickened and hardened bowel wall of the descending colon, sigmoid colon and rectum and board-like mesentery, whereas the remaining bowel was normal. An open

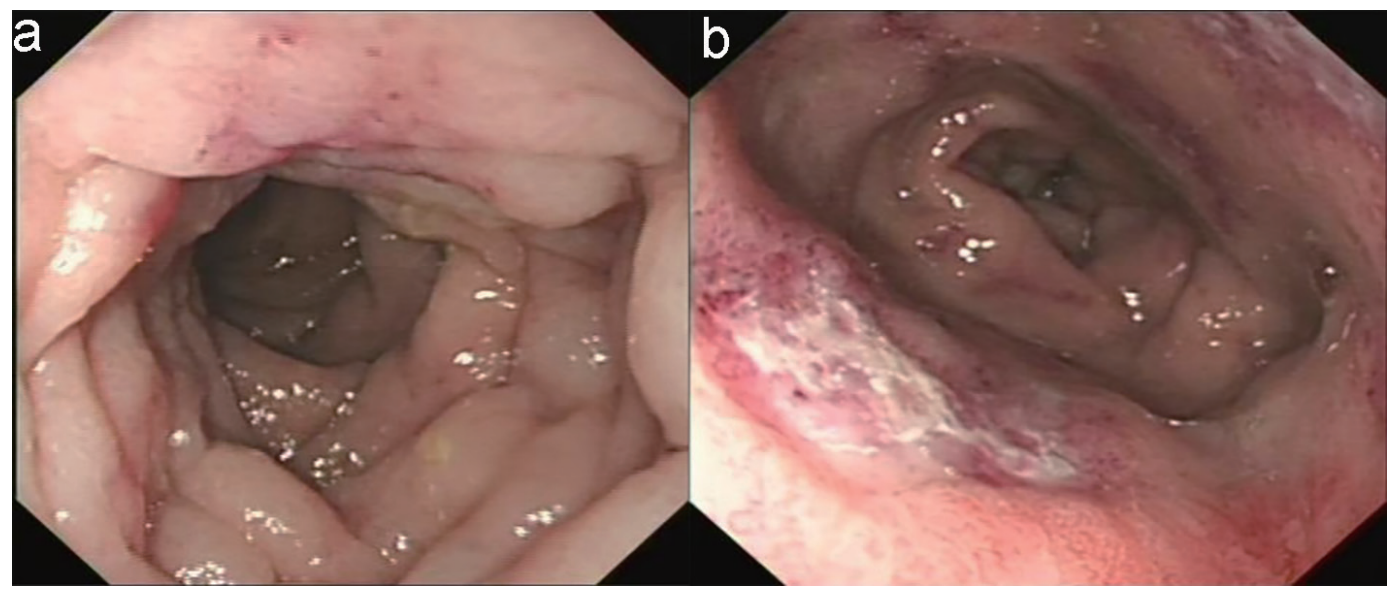

Figure 2. Colonoscopy revealed that the descending colon mucosa had hyperemia, constriction, edema, and with cobble-stone sign (a) and the rectum was the most seriously affected segment which had circumferential erosion and superficial ulcer (b). 


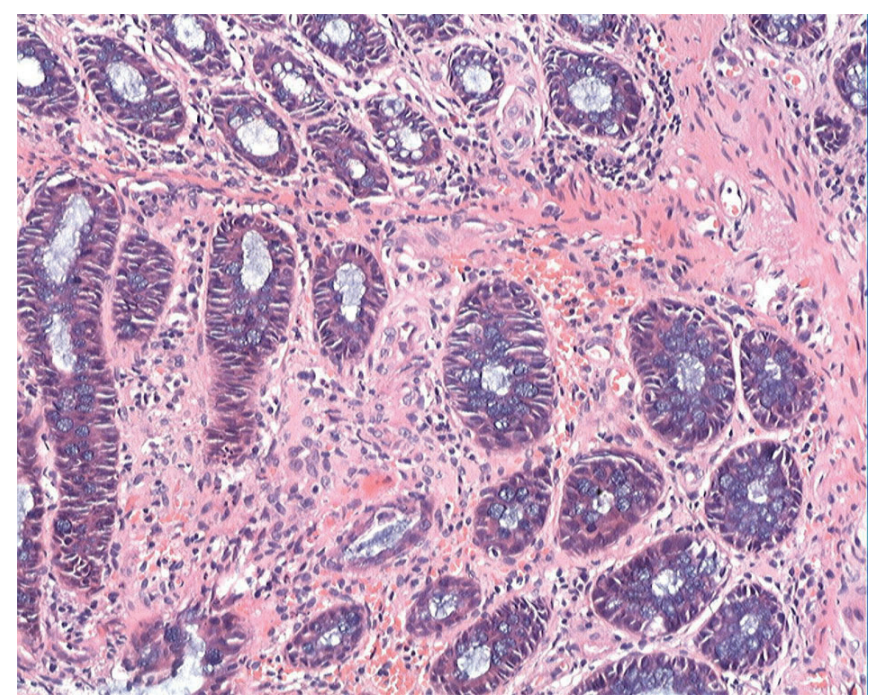

Figure 3. Colonic biopsy demonstrated mucosal infiltration by inflammatory cells and focal interstitial fibrosis (H\&E staining, $\times 20$ ).

laparotomy was performed with resection of the left colon and rectum, creation of a Hartman pouch and creation of transverse colon ostomy.

Macroscopically, the resected bowel segment was $48 \mathrm{~cm}$ long, and the resected mesentery was $48 \times 7 \times 6 \mathrm{~cm}$ in size which was stiff (Fig. 7). Microscopically, there were fat necrosis, multifocal lipid-filled macrophages, lymphocytes and multifocal fibrosis in the mesentery adipose tissue. Submucosal was markedly edematous. Mucosal erosions and hemorrhage were present. However, there were no granulomas, vasculitis or malignancy (Fig. 8). Histopathology examination confirmed a diagnosis of sclerosing mesenteritis with a prominence of fibrosis, i.e., retractile mesenteritis. After the surgery, the patient had annual surveillance for three times without recurrence of symptoms. Contrast-enhanced CT and colonoscopy expounded the postoperation changes without any other lesion.

\section{Discussion}

Our patient presented with progressive dyschezia, abdominal pain and abdominal mass in the left lower quadrant and weight

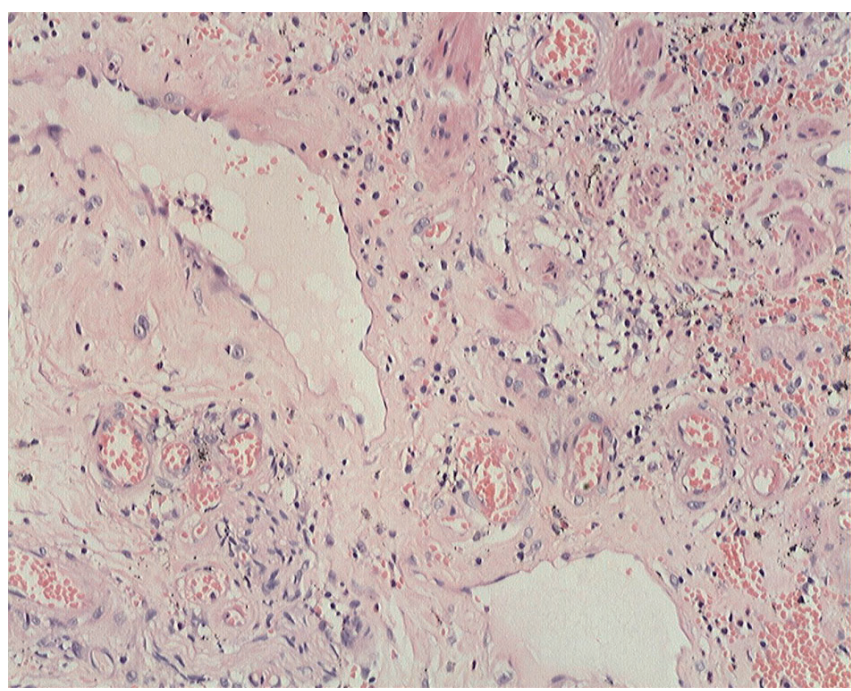

Figure 5. ESD specimen showed erosion, inflammatory exudates, granulation tissue, and fibrinoid degeneration of small blood vessel wall (H\&E staining, $\times 20$ ).

loss within months. The patient's CT exhibited increased thickness of the involved mesentery, enlarged lymph nodes and strand-like densities around the mesenteric vessels. The patient was initially treated with oral mesalazine (2.0 g QID) and intravenous dexamethasone $(10 \mathrm{mg}$ QD $\times 3$ days $)$, but without any improvement. Although the preoperative laboratory results, colonoscopy and PET-CT findings indicated inflammatory diseases, a specific disease could not be diagnosed. The final diagnosis of SM was only made based on pathologic findings in the partial colectomy, which included fat necrosis, multifocal lipid-filled macrophages, lymphocytes and multifocal fibrosis in the mesentery adipose tissue. SM is a rare disease of uncertain etiology characterized by chronic non-specific inflammation, fat necrosis and fibrosis involving adipose tissue of the mesentery [1]. Purported risk factors include autoimmune disorders, infection (i.e. Mycobacterium tuberculosis), trauma (i.e. abdominal surgery), mesenteric ischemia as well as both solid and hematologic malignancies. It is also thought to be related to rheumatologic diseases (rheumatoid arthritis, ankylosing spondylitis and retroperitoneal fibrosis) [1]. SM was reported to be closely related to IgG4-related disease

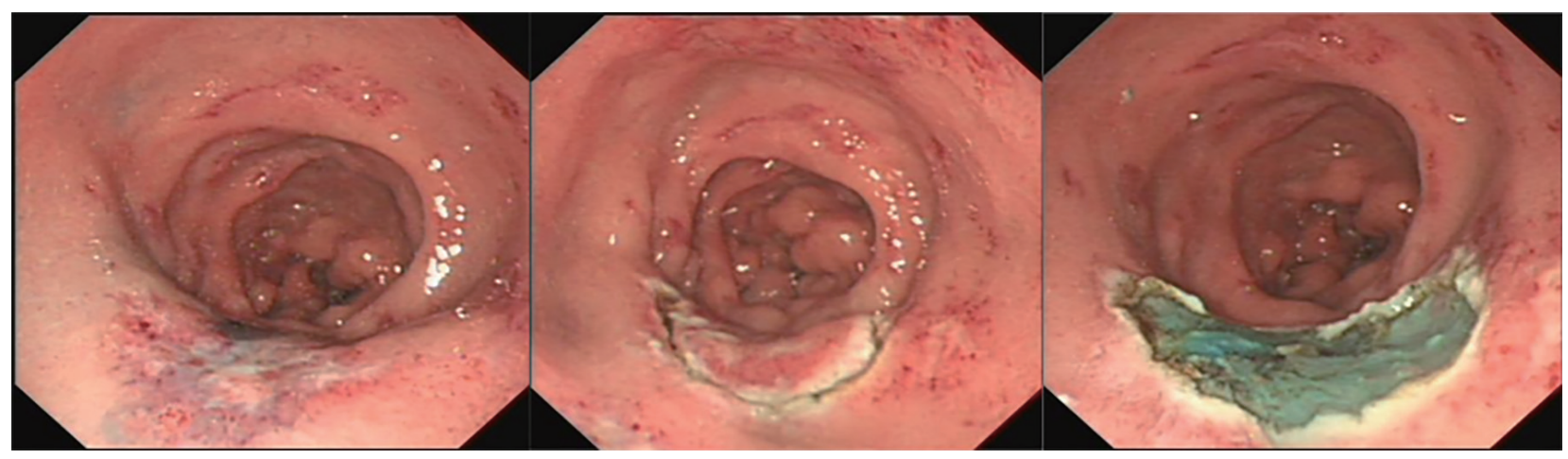

Figure 4. Endoscopic submucosal dissection procedure. 


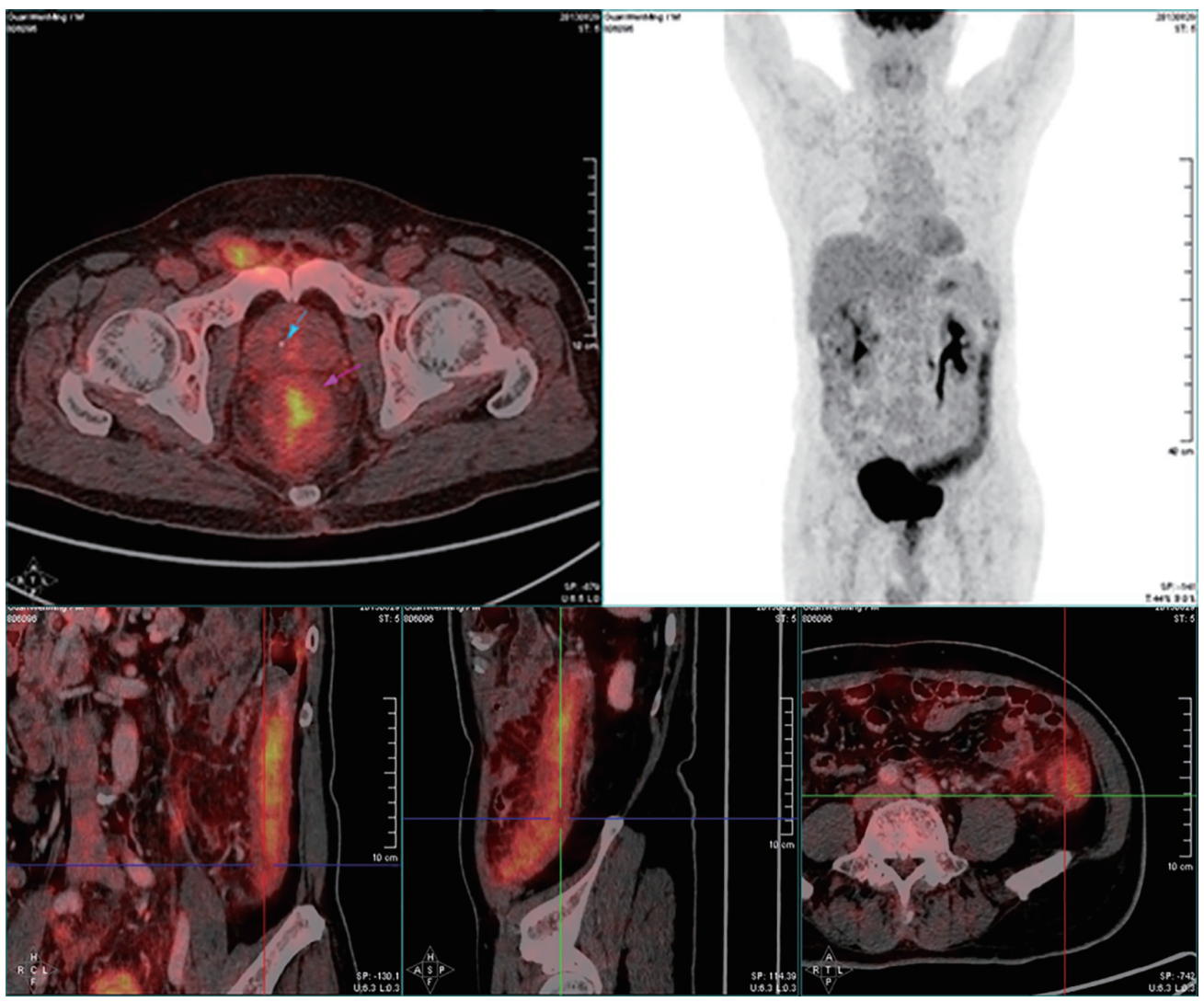

Figure 6. PET-CT of the abdomen showed significant accumulation of FDG uptake (SUV max: 5.7), diffuse edema and thickening (about $1.5 \mathrm{~cm}$ ) of the distal descending colon wall, indicating an inflammatory lesion.

(IgG4-RD) [3].

The exact etiology of SM remains unknown. SM often affects the middle-aged and older adults with the male predominance [4]. It usually involves the mesentery of the small bowel, appendix, mesoappendix and, less frequently, the sigmoid colon or other intraabdominal fatty tissue. Macroscopically, the thickened mesentery can be presented in three types: 1) diffuse thickening of the mesentery; 2) single knotty thickening at the root of the mesentery; and 3) multiple knotty thickenings of the mesentery. Microscopically, SM has a mixed pathologic change of fat necrosis, chronic inflammation and fibrosis of the mesentery. According to dominant pathological changes, the following terminologies have been used: mesenteric panniculitis (chronic inflammation and fibrosis), mesenteric lipod-
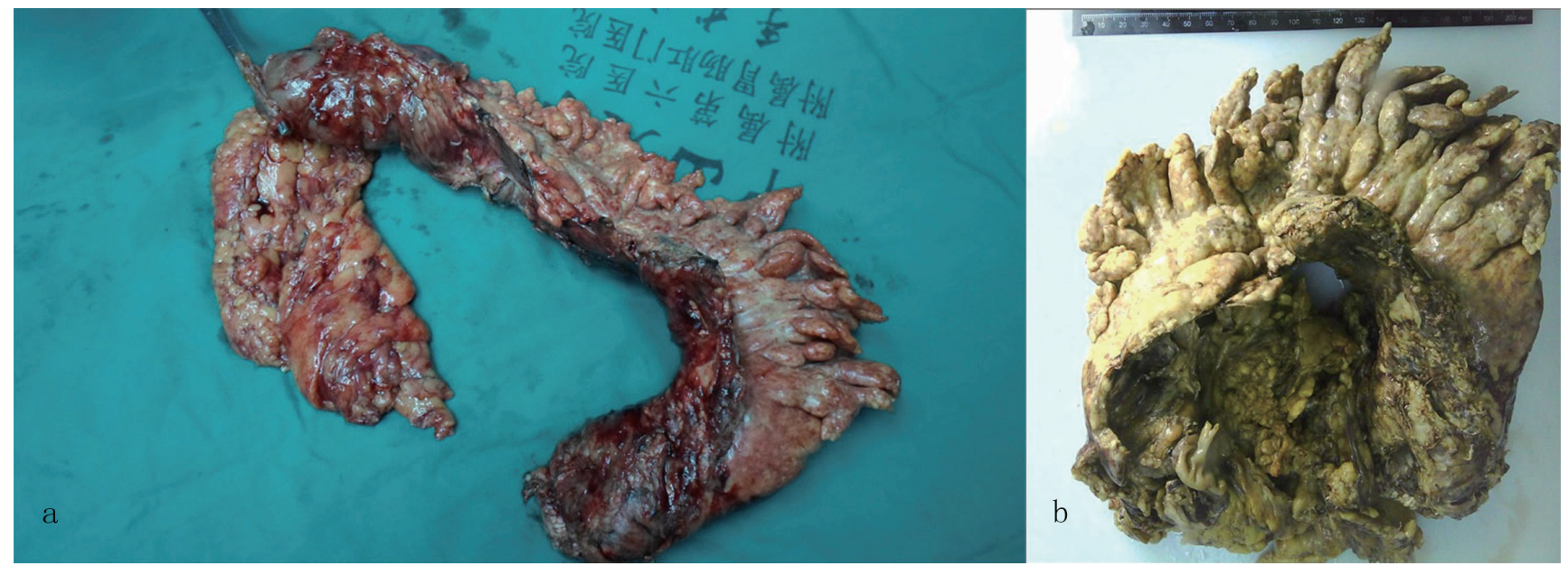

Figure 7. Macroscopic examination of the partial colectomy revealed (a) a $48 \mathrm{~cm}$ segment of stiff bowel and (b) a $48 \times 7 \times 6 \mathrm{~cm}$ portion of stiff mesentery. 


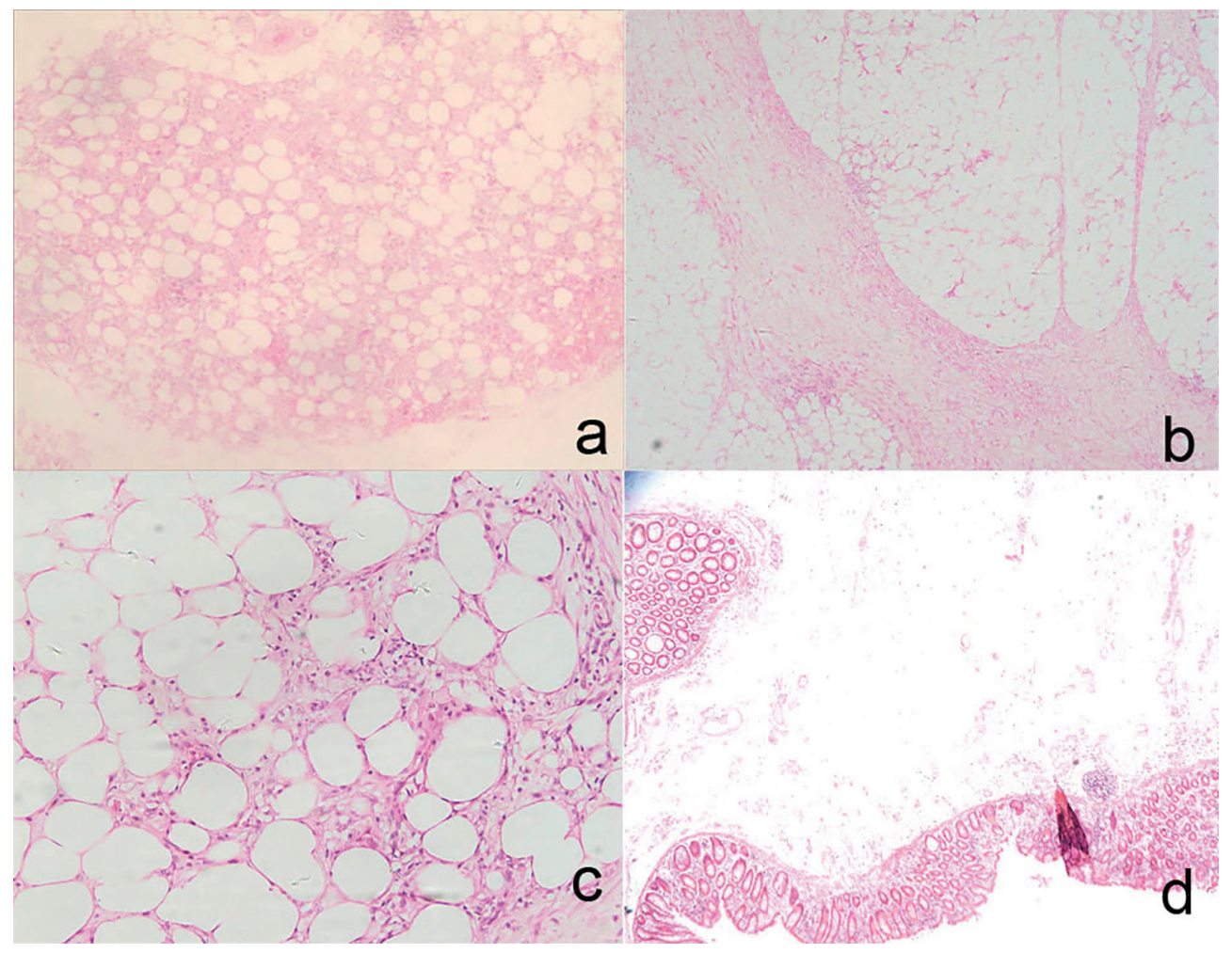

Figure 8. Histologic findings in the partial colectomy and mesentery included fat necrosis and multifocality lipid-filled macrophages in mesentery adipose tissue (H\&E staining, $\times 4, a$ ), multifocality fibrosis in mesentery adipose tissue (H\&E staining, $\times 4, b$; H\&E staining, $\times 20, c)$, markedly edematous submucosa and partially eroded and hemorrhagic mucosa (H\&E staining, $\times 2.5, d)$.

ystrophy (fat necrosis) and retractile mesenteritis (fibrosis) [1]. Patients with SM may be asymptomatic or may present with abdominal discomfort, nausea, vomiting, diarrhea, constipation or abdominal mass [1]. Laboratory findings were non-specific and may include elevation in erythrocyte sedimentation rate and anemia. The best non-invasive method to diagnose $\mathrm{SM}$ is CT [5]. SM shows $0.6 \%$ prevalence in patients who undergo abdominal CT. Common CT findings include increased thickness of the involved mesentery, increased fat density due to infiltration by inflammatory cells, fibrosis and enlarged lymph nodes, fat ring sign surrounding the mesenteric vessels, pseudocapsule of a peripheral band delineating the inflammatory mesenteric mass from the surrounding normal folds, dilated or engorged mesenteric vessels, well-defined or poorlydefined mesenteric mass (usually small bowel mesentery) with displacement of the bowel loops, strand-like densities around the mesenteric vessels, and well-defined soft tissue nodules usually less than $5 \mathrm{~mm}$ (Table 1) [5]. Endoscopic features of the intestinal mucosa within the bowel segment involved by SM are often non-specific resembling ischemic colitis or normal because the pathological changes in SM mainly involve the mesenteric and submucosal fat of the bowel with the mucosa remaining intact. SM is an uncommon disease in the Chinese population; hence the therapeutic strategies are empirical. Previous research has shown that symptomatic patients may benefit from medical therapy, particularly tamoxifen and prednisone combination treatment. Surgery is required in some situation, such as intestinal obstruction and catalepsy [1].
In conclusion, SM, a rare disease, should be considered in the differential diagnosis in malignancy and inflammatory disease of the intestine. Imaging studies, preferably CT, of the abdomen, including the bowel and mesentery, should be performed. Finally, surgery, intraoperative findings and pathologic examination of the resected bowel segment and mesentery can confirm the diagnosis of SM. Although several drugs (steroids, colchicine, immunosuppressive agents, or orally administered progesterone) may be used to treat SM, surgery is preferred for those patients who are at the stage of fibrosis (retractile mesenteritis), particularly in those patients complicated by bowel obstruction. Our case report illustrates the use of multidiscipli-

\section{Table 1. Radiographic Features of Sclerosing Mesenteritis}

Thickness of the involved mesentery

Increased fat density

Fibrosis and enlarged lymph nodes

Fat ring sign

Pseudocapsule

Dilated or engorged mesenteric vessels

Well-defined or poorly defined mesenteric mass

Strand-like densities around the mesenteric vessels

Well-defined soft tissue nodules (usually less than $5 \mathrm{~mm}$ )

Bowel obstruction 
nary approach to diagnose SM.

\section{Ethics Approval}

This case report was approved by the Institutional Review Board of the Sixth Affiliated Hospital of Sun Yat-sen University.

\section{Consent}

The patient gave informed consent for patient's data acquisition and signed the Sixth Affiliated Hospital of Sun Yat-sen University informed consent for patient's data acquisition.

\section{Competing Interests}

The authors declare that they have no competing interests.

\section{Funding}

The case report was supported by Sun Yat-Sen University Clinical Research 5010 Program (Item Number: 2104008).

\section{Author Contributions}

Huan He, Min Zhi, Min Zhang, Mingli Su, and Huangwei Chen analyzed and interpreted the patient data regarding the Sclerosing Mesenteritis. Huan He was a major contributor in writing the manuscript. Min Zhi performed the endoscopy examination of the patient. Huang Yan performed the histological examination of the bowel tissue. Zhiyang Zhou performed the radiographic examination of the patient. Liang Kang and Jiangping Wang performed the surgery of the patient. Xiang Gao was involved in administrative. Pin-jin $\mathrm{Hu}$ was involved in study supervision. All authors read and approved the final manuscript.

\section{Abbreviations}

SM: sclerosing mesenteritis; CT: computed tomography; $\mathrm{Hb}$ : hemoglobin; CRP: C-reactive protein; IBD: inflammatory bowel disease; PE: physical examination; ESD: endoscopic submucosal dissection; PET: positron emission tomography

\section{References}

1. Hussein MR, Abdelwahed SR. Mesenteric panniculitis: an update. Expert Rev Gastroenterol Hepatol. 2015;9(1):6778.

2. Ogden WW, 2nd, Bradburn DM, Rives JD. Mesenteric Panniculitis: Review of 27 Cases. Ann Surg. 1965; 161:864-875.

3. Abe A, Manabe T, Takizawa N, Ueki T, Yamada D, Nagayoshi K, Sadakari Y, et al. IgG4-related sclerosing mesenteritis causing bowel obstruction: a case report. Surg Case Rep. 2016;2(1):120.

4. Emory TS, Monihan JM, Carr NJ, Sobin LH. Sclerosing mesenteritis, mesenteric panniculitis and mesenteric lipodystrophy: a single entity? Am J Surg Pathol. 1997;21(4):392-398.

5. Daskalogiannaki M, Voloudaki A, Prassopoulos P, Magkanas E, Stefanaki K, Apostolaki E, Gourtsoyiannis N. CT evaluation of mesenteric panniculitis: prevalence and associated diseases. AJR Am J Roentgenol. 2000;174(2):427-431. 